\title{
Endoscopic system for automated high dynamic range inspection of moving periodic structures (Withdrawal Notice)
}

Cornelius Hahlweg, Hendrik Rothe

Cornelius Hahlweg, Hendrik Rothe, "Endoscopic system for automated high dynamic range inspection of moving periodic structures (Withdrawal Notice)," Proc. SPIE 9579, Novel Optical Systems Design and Optimization XVIII, 957908 (3 September 2015); doi: 10.1117/12.2188726

SPIE Event: SPIE Optical Engineering + Applications, 2015, San Diego, California, United States 


\section{Endoscopic system for automated high dynamic range inspection of moving periodic structures (Withdrawal Notice)}

Cornelius Hahlweg, bbw Hochscule (Germany) and Hendrik Rothe, Helmut-Schmidt University (Germany)

Proc. SPIE 9579, Novel Optical Systems Design and Optimization XVIII, 957908 (September 3, 2015); doi: 10.1117/12.2188726

Online Publication Date: 3 September 2015

Withdrawn from Publication: 27 October 2016

Conference Date: 12-14 August, 2015

Conference Location: San Diego, California, United States

Conference Title: Novel Optical Systems Design and Optimization XVIII

Conference Chairs: G. Groot Gregory, Arthur J. Davis, Cornelius F. Hahlweg

This paper, originally published on 3 September, 2015, was withdrawn on 27 October, 2016, at the authors' request. 